\title{
Accurate and Simple Estimator for Lossy Wave Equation
}

\author{
H. C. So, Senior Member, IEEE, Md. Tawfiq Amin, and Frankie K. W. Chan
}

\begin{abstract}
In this letter, parameter estimation of a uniformly sampled signal that satisfies the lossy wave equation in Gaussian noise is investigated. By exploiting the linear prediction property of the noise-free signal, a maximum likelihood estimator for the parameters is first developed. Relaxation is then applied to yield a simple and accurate algorithm. It is shown that the estimation performance of the proposed method attains Cramér-Rao lower bound.
\end{abstract}

Index Terms-Linear prediction, maximum likelihood estimator, parameter estimation, wave equation.

\section{INTRODUCTION}

C ONSIDER the one-dimensional wave equation:

$$
\frac{\partial^{2} u(x, t)}{\partial x^{2}}-\frac{1}{c^{2}} \frac{\partial^{2} u(x, t)}{\partial t^{2}}=0
$$

where $u(x, t)$ is a measured phenomenon at location $x$ and time $t$ that satisfies (1) and $c$ is the wave propagation speed. It is well known that the general solution of Fourier transform of $u(x, t)$, denoted by $U(x)$, is of the form

$$
U(x)=A e^{j k x}+B e^{-j k x}
$$

where $k$ is the unknown wave number while $A$ and $B$ are unknown complex constants determined by the auxiliary or boundary conditions. As (2) is a realistic model of the physical phenomenon in many scenarios [1] such as describing the voltage along a transmission line and medical imaging modality of elastography, it is of interest to find the complex-valued parameters, namely, $k, A$ and $B$.

In [1], the nonlinear least squares (NLS) algorithm is proposed to find the parameters of interest given a sampled and noisy version of (2). Although the NLS estimator can attain the maximum likelihood (ML) performance under white Gaussian noise environment, it is hard to implement in practice as its objective function is multi-modal. Noting that (2) corresponds to the sum of two exponentially damped sinusoids, an alternative approach is to utilize its linear prediction (LP) property [2] and apply the iterative quadratic maximum likelihood (IQML) technique [3], [4] to solve for $k$ first, and $A$ and $B$ are then esti-

Manuscript received April 01, 2009; revised May 24, 2009. First published June 10, 2009; current version published July 09, 2009. This work was supported by a grant from CityU (Project 7002499). The associate editor coordinating the review of this manuscript and approving it for publication was Prof. Markku Renfors.

The authors are with Department of Electronic Engineering, City University of Hong Kong.

Color versions of one or more of the figures in this paper are available online at http://ieeexplore.ieee.org.

Digital Object Identifier 10.1109/LSP.2009.2024797 mated accordingly. In this letter, we show that the LP structure of (2) can be further simplified with the use of the conjugate nature in the exponential terms. Based on the IQML approach, a different ML estimator is developed and relaxation is then made to produce a computationally attractive algorithm with a global solution. Computer simulation shows that the estimation performance of the proposed relaxation method can attain the minimum variance bound, namely, Cramér-Rao lower bound (CRLB) [1] and is superior to the NLS and standard IQML estimators in terms of threshold performance.

\section{Algorithm DeVElopment}

The discrete-time model for the uniformly sampled signal that satisfies (2) in additive noise is

$$
R(n)=U(n)+Q(n), \quad n=1,2 \ldots, N .
$$

For simplicity but without loss of generality, unity sampling interval is assumed and $Q(n)$ is a zero-mean white complex Gaussian process with unknown variance $\sigma^{2}$. Given the $N$ measurements of $R(n), 1,2 \ldots, N$, the objective is to find $k, A$ and $B$.

As $U(n)$ consists of two exponentially damped sinusoids, it will follow the LP property:

$$
U(n)+b_{1} U(n-1)+b_{2} U(n-2)=0
$$

where $k$ is determined from the roots of $z^{2}+b_{1} z+b_{2}=0[2]$. With the use of (2), (4) is expanded as:

$$
\begin{aligned}
A e^{j k n}\left(1+b_{1} e^{-j k}\right. & \left.+b_{2} e^{-2 j k}\right) \\
& +B e^{-j k n}\left(1+b_{1} e^{j k}+b_{2} e^{2 j k}\right)=0 .
\end{aligned}
$$

As (5) holds for all values of $A$ and $B$, we then have two equations, namely, $1+b_{1} e^{-j k}+b_{2} e^{-2 j k}=0$ and $1+b_{1} e^{j k}+$ $b_{2} e^{2 j k}=0$. Solving them yields $b_{1}=-2 \cos (k)$ and $b_{2}=1$, and thus (4) can be simplified as

$$
U(n)-2 \cos (k) U(n-1)+U(n-2)=0
$$

which corresponds to a very simple LP relation as in the case of a single real tone but now $\cos (k)$ is a complex number. As in conventional sinusoidal parameter estimation, finding $k$ is the first and crucial step because it is a nonlinear function in the received data sequence. The remaining parameters, namely, $A$ and $B$, can then be estimated in a more straightforward manner after its determination.

Let $\lambda=2 \cos (k)$ and define $\mathbf{r}_{i}=[R(i), R(i+1), \ldots R(i+$ $N-3)]^{T}, i=1,2,3$, where ${ }^{T}$ denotes the transpose operator. Using (6) and following the development in [5], it is shown that the ML estimate for $\lambda$, denoted by $\hat{\lambda}$, in the presence of Gaussian 
noise, can be determined from the minimization problem shown in (7) at the bottom of the page. where $\tilde{\lambda}$ is the optimization variable for $\lambda,{ }^{H}$ and ${ }^{-1}$ represent the conjugate transpose and matrix inverse operations, respectively. The covariance matrix $\boldsymbol{\Sigma}$ is also a function of $\tilde{\lambda}$ and has the form of $E\left\{\mathbf{p} \mathbf{p}^{H}\right\}$ where $E$ is the expectation operator, with $\mathbf{p}=[P(1), P(2), \ldots, P(N-$ $2)]^{T}$ whose element is $P(n)=Q(n+2)-\tilde{\lambda} Q(n+1)+Q(n)$, $n=1,2, \ldots, N-2$. For zero-mean white Gaussian noise, $\Sigma$ has the form of (8), shown at the bottom of the page, where Toeplitz denotes Toeplitz operator, ${ }^{*}$ is the conjugate operator and $\sigma^{2}$ is ignored as it has no effect on the minimization of (7). It is noteworthy that this ML estimator can be extended to more general Gaussian process of $Q(n)$ as long as $\boldsymbol{\Sigma}$ is known up to a scalar. As in the NLS estimator of [1], the objective function in (7) is also multi-modal, which means that it is not guaranteed to find the globally optimum point.

Utilizing the idea of the IQML technique [3], [4], we relax (7) into a quadratic function by considering $\Sigma$ is independent of $\tilde{\lambda}$ so that global optimization is attained and the estimate of $\lambda$, denoted by $\hat{\lambda}$, is easily computed as:

$$
\hat{\lambda}=\left(\mathbf{r}_{2}^{H} \boldsymbol{\Sigma}^{-1} \mathbf{r}_{2}\right)^{-1} \mathbf{r}_{2}^{H} \boldsymbol{\Sigma}^{-1}\left(\mathbf{r}_{3}+\mathbf{r}_{1}\right) .
$$

The estimated $\lambda$ is then employed to update $\boldsymbol{\Sigma}$. We repeat the above steps for a few times with an initial guess of $\boldsymbol{\Sigma}$. The iterative procedure of the proposed estimator for $k$ is summarized as follows.

a) Set $\boldsymbol{\Sigma}$ to be the identity matrix.

b) Compute $\hat{\lambda}$ using (9).

c) Use $\hat{\lambda}$ to construct $\boldsymbol{\Sigma}$ of (8).

d) Repeat Steps (ii) and (iii) for $I$ times until parameter convergence.

e) (v) Use the finalized $\hat{\lambda}$ to compute the estimate of $k$ as $\hat{k}=\cos ^{-1}(\hat{\lambda} / 2)$.

When the initial estimate of $\lambda$ is sufficiently accurate, it is expected that global convergence is attained. To derive the convergence properties of the relaxation algorithm, the interested reader is referred to [6]. Employing $\hat{k}, A$ and $B$ are then solved by minimizing the following least squares (LS) cost function:

$$
\sum_{n=1}^{N}\left|R(n)-\tilde{A} e^{j \hat{k} n}-\tilde{B} e^{-j \hat{k} n}\right|^{2}
$$

where $\tilde{A}$ and $\tilde{B}$ are the corresponding optimization variables. Differentiating (10) with respect to $\tilde{A}$ and $\tilde{B}$ and setting the resultant expressions to zero, the LS estimates of $A$ and $B$, denoted by $\hat{A}$ and $\hat{B}$, are computed as

$$
\left[\begin{array}{c}
\hat{A} \\
\hat{B}
\end{array}\right]=\left(\mathbf{G}^{H} \mathbf{G}\right)^{-1} \mathbf{G}^{H} \mathbf{r}
$$

where

$$
\begin{aligned}
\mathbf{G} & =\left[\begin{array}{cc}
e^{j \hat{k}} & e^{-j \hat{k}} \\
\vdots & \vdots \\
e^{j \hat{\hat{k}} N} & e^{-j \hat{k} N}
\end{array}\right] \\
& \text { and } \\
\mathbf{r} & =\left[\begin{array}{lll}
R(1) & \cdots & R(N)
\end{array}\right]^{T} .
\end{aligned}
$$

We expect that $\hat{A}$ and $\hat{B}$ are optimum estimates as long as $\hat{k}$ is optimum because the disturbance in (10) is white.

\section{Simulation Results}

Computer simulations have been carried out to evaluate the parameter estimation performance of the proposed algorithm for lossy wave equation in zero-mean white complex Gaussian noise. The mean square errors (MSEs), namely, $E\left\{|\hat{k}-k|^{2}\right\}$, $E\left\{|\hat{A}-A|^{2}\right\}$ and $E\left\{|\hat{B}-B|^{2}\right\}$, are employed as the performance measures. The NLS [1] and standard IQML [2] methods as well as the corresponding minimum variance bounds, namely, CRLBs, are used for comparison. We examine $I=1,2,3$ in the proposed relaxation method as no obvious improvement is observed for more iterations. On the other hand, the NLS algorithm is realized by the Newton's method with the true values as initial estimates. For the IQML algorithm based on the LP model of (4), five iterations are employed. There are two roots in $z^{2}+b_{1} z+b_{2}=0$ but we notice that only one of them is valid, and the following procedure is devised to select the correct one. Two $\hat{k}$ are computed from the two roots and the corresponding estimates of $A$ and $B$ are then determined using (11). The IQML estimates are given by the parameter set which produces the smaller value of (10). We scale $Q(n)$ to produce different signal-to-noise ratio (SNR) condition where SNR = $\sum_{n=1}^{N}|U(n)|^{2} /\left(N \sigma^{2}\right)$. Unless stated otherwise, the parameters of $U(n)$ are $k=0.05+j 0.28, A=1+j 0.6$ and $B=$

$$
\begin{aligned}
\hat{\lambda} & =\arg \min _{\tilde{\lambda}}\left(\mathbf{r}_{3}-\tilde{\lambda} \mathbf{r}_{2}+\mathbf{r}_{1}\right)^{H} \boldsymbol{\Sigma}^{-1}\left(\mathbf{r}_{3}-\tilde{\lambda} \mathbf{r}_{2}+\mathbf{r}_{1}\right) \\
\boldsymbol{\Sigma} & =\operatorname{Toeplitz}\left(\left[2+|\tilde{\lambda}|^{2},-\tilde{\lambda}-\tilde{\lambda}^{*}, 1,0, \ldots, 0\right]\right) \\
& =\left[\begin{array}{cccccccc}
2+|\tilde{\lambda}|^{2} & -\tilde{\lambda}-\tilde{\lambda}^{*} & 1 & 0 & 0 & 0 & \ldots & 0 \\
-\tilde{\lambda}-\tilde{\lambda}^{*} & 2+|\tilde{\lambda}|^{2} & -\tilde{\lambda}-\tilde{\lambda}^{*} & 1 & 0 & 0 & \ldots & 0 \\
1 & -\tilde{\lambda}-\tilde{\lambda}^{*} & 2+|\tilde{\lambda}|^{2} & -\tilde{\lambda}-\tilde{\lambda}^{*} & 1 & 0 & \ldots & 0 \\
\vdots & \vdots & \vdots & \vdots & \vdots & \vdots & \vdots & \vdots \\
0 & \ldots & \ldots & 0 & 1 & -\tilde{\lambda}-\tilde{\lambda}^{*} & 2+|\tilde{\lambda}|^{2} & -\tilde{\lambda}-\tilde{\lambda}^{*} \\
0 & \ldots & \ldots & \ldots & 0 & 1 & -\tilde{\lambda}-\tilde{\lambda}^{*} & 2+|\tilde{\lambda}|^{2}
\end{array}\right]
\end{aligned}
$$




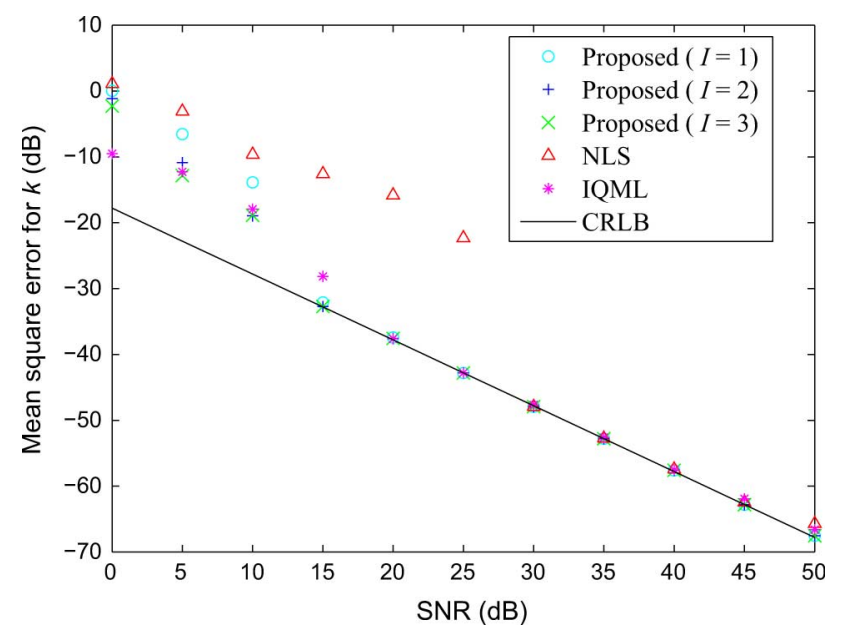

Fig. 1. Mean square error for $k$ versus SNR.

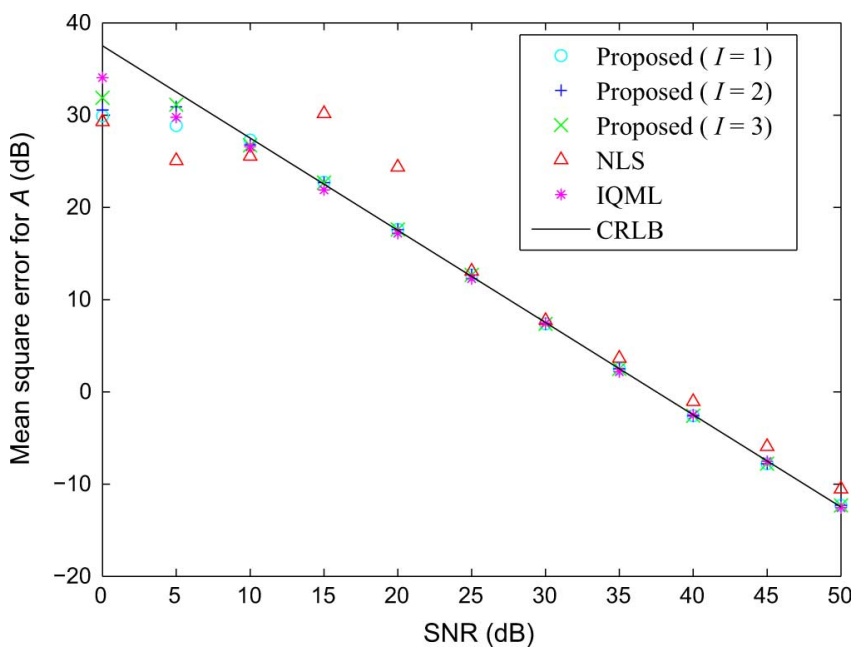

Fig. 2. Mean square error for $A$ versus SNR.

$-0.6+j 0.7$ while a data length of $N=20$ is assigned. All results provided are averages of 1000 independent runs.

In the first test, the parameter estimation performance versus SNR is examined. Figs. 1-3 show the MSEs of $\hat{k}, \hat{A}$ and $\hat{B}$ versus SNR, respectively. In our method, the accuracy of all parameters of interest attains the corresponding CRLBs for SNR $\in[15,50] \mathrm{dB}$. Although the NLS and IQML methods also give optimum performance for higher SNRs, the proposed scheme is superior to them as it has a smaller threshold SNR. Furthermore, we observe that the difference between $I=2$ and $I=3$ in our algorithm is negligible. Note that all methods become biased estimators for SNR $<10 \mathrm{~dB}$ as their MSEs for $A$ are below the performance limits.

In the second test, the average performance for different values of $k$ is studied. In fact, the real and imaginary parts of $k$ correspond to the frequency and damping factor of a damped sinusoid, and according to [1], their typical ranges are $(0, \pi)$ and $(0,1)$, respectively. As $A$ and $B$ are nuisance parameters which are obtained after estimating $k$, their randomization is not considered. In Fig. 4, the real part of $k$ is uniformly drawn from $[0.01 \pi, 0.99 \pi]$ in each independent trial, while in Fig. 5, the imaginary component of $k$ is a uniform number drawn from

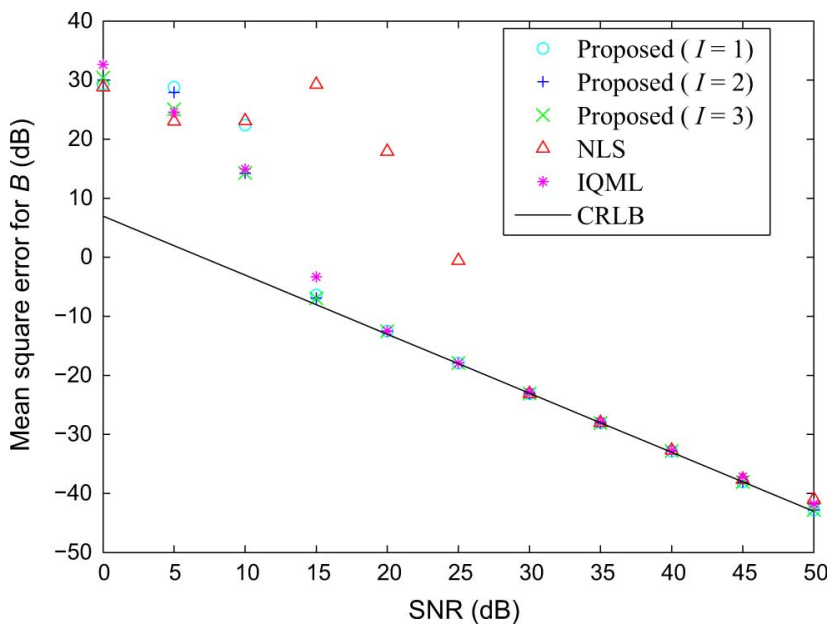

Fig. 3. Mean square error for $B$ versus SNR.

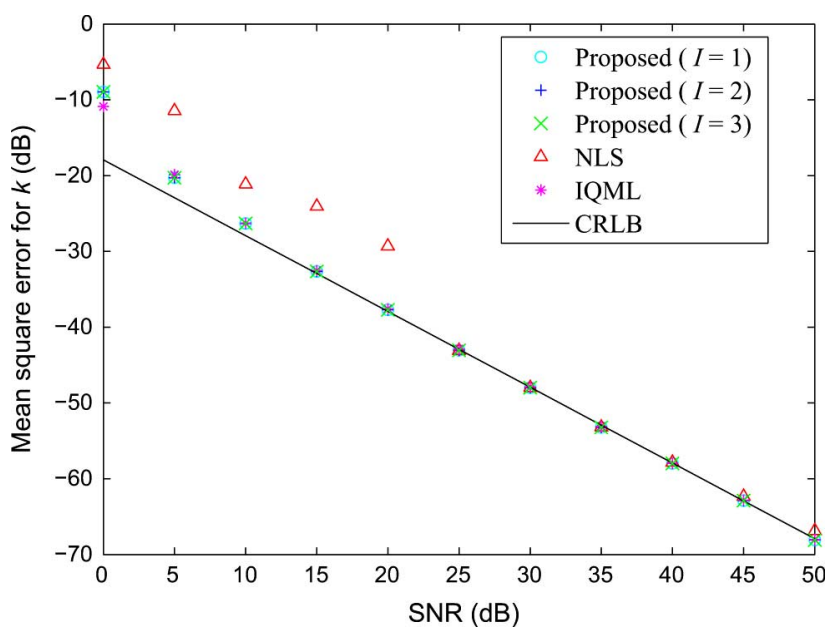

Fig. 4. Average performance for $k$ versus SNR when its real part is randomized.

$[0,1]$ in each run. The findings from Figs. 4 and 5 are similar to those of the first test. Notice that the results for $A$ and $B$ have not been included as similar observations are obtained.

Finally, the effect of $N$ on the accuracy is investigated. Fig. 6 shows the MSEs of $\hat{k}$ for $N \in[5,30]$. It is seen that the proposed estimator outperforms the other two as it gives optimum performance for $N \in[10,30]$, although optimality is not attained at $N=5$. Again, the results for $A$ and $B$ have not been included as similar observations are obtained. Based on the simulation study, we believe that the initial estimate of $k$ is crucial for obtaining the global solution, that is, global convergence will be attained if it is sufficiently close to the global minimum of (7). When the data length is too short and/or the SNR is too small, it is very likely that the initial estimate of is far from the global minimum which results in local convergence.

\section{CONCLUSION}

An accurate and computationally simple estimator for parameters of the lossy wave equation in additive white complex Gaussian noise is devised. The key ideas are to utilize the signal linear prediction property and relax the corresponding maximum likelihood estimator. It is demonstrated that the 


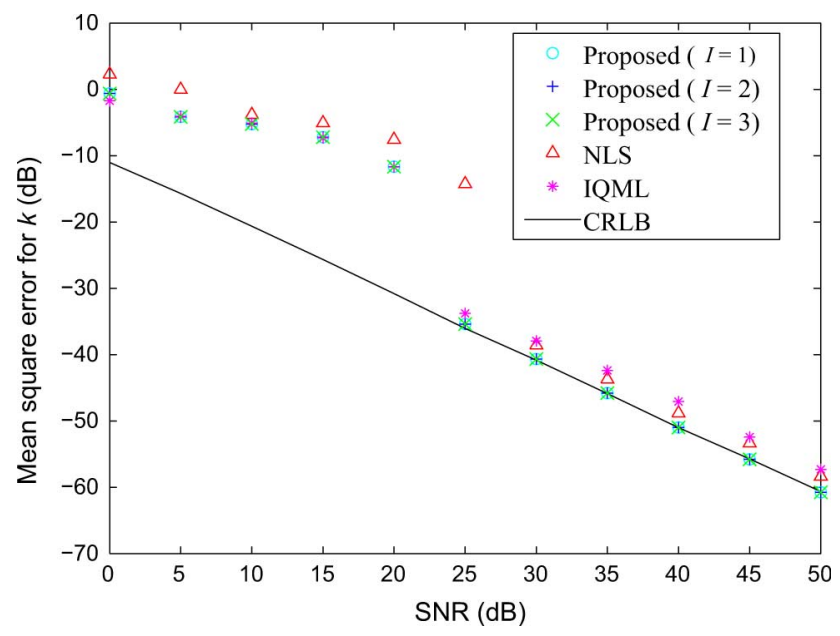

Fig. 5. Average performance for $k$ versus SNR when its imaginary part is randomized.

estimation performance of the proposed algorithm can attain Cramér-Rao lower bound and outperform the nonlinear least squares and standard iterative quadratic maximum likelihood methods. A possible future direction is to extend the algorithm development to higher-dimensional spaces by using Green's functions to find the implicit relationship similar to (6), although boundary conditions and sampling issues will significantly complicate the study.

\section{ACKNOWLEDGMENT}

The authors would like to thank the anonymous reviewers for their useful comments and suggestions.

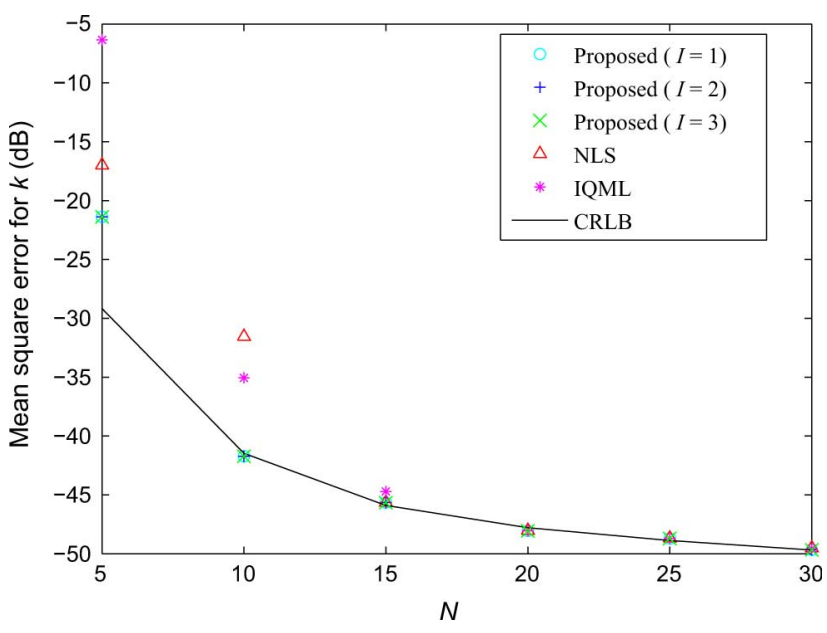

Fig. 6. Mean square error for $k$ versus $N$.

\section{REFERENCES}

[1] T. E. Oliphant, "On parameter estimates for the lossy wave equation," IEEE Trans. Signal Process., vol. 56, pp. 49-60, Jan. 2008.

[2] G. Zhu and Y. Hua, "Quantitative NMR signal analysis by an iterative quadratic maximum likelihood method," Chem. Phys. Lett., vol. 264, pp. 424-428, Jan. 1997.

[3] R. Kumaresan, L. L. Scharf, and A. K. Shaw, "An algorithm for pole-zero modeling and spectral analysis," IEEE Trans. Acoust., Speech Signal Process., vol. ASSP-34, pp. 637-640, Jun. 1986.

[4] Y. Bresler and A. Macovski, "Exact maximum likelihood parameter estimation of superimposed exponential signals in noise," IEEE Trans. Acoust., Speech. Signal Process., vol. ASSP-34, pp. 1081-1089, Oct. 1986.

[5] M. Aoki and P. C. Yue, "On a priori error estimates of some identification methods," IEEE Trans. Automat. Contr., vol. 15, pp. 541-548, Oct. 1970.

[6] J. Li, P. Stoica, and Z. Liu, "Comparative study of IQML and MODE direction-of-arrival estimators," IEEE Trans. Signal Process., vol. 46, pp. 149-160, Jan. 1998. 